CONCURSO ESTUDIANTIL DE DISEÑO

\title{
HIPO: Aplicación multiplataforma para el apoyo del aprendizaje de habilidades matemáticas dirigida a personas con discapacidad intelectual
}

\author{
Abies C. Durán L., Mario A. Marín B., Karla N. \\ Rocha R., Julián Cárdenas P., Aída L. González L.
}

Publicado: 31 Octubre 2018

\begin{abstract}
Resumen
El desarrollo de habilidades matemáticas de personas con discapacidad intelectual es importante, ya que les permite participar responsablemente en la dinámica familiar y social además de lograr la inclusión. En este trabajo se expone el desarrollo de una aplicación computacional de apoyo para el aprendizaje de habilidades matemáticas con base en un programa curricular desarrollado en una institución de educación especial. Se utilizó una Metodología de Diseño Centrado en el Usuario en este proyecto además de Diseño Participativo; se realizó el análisis de contexto de uso y requerimientos mediante observaciones de campo, en la fase de diseño se realizaron prototipos de baja y alta fidelidad y se implementó un diseño plano; en la fase de evaluación además de pruebas de usuarios fue necesario aplicar pruebas con seguidor ocular. Realizando las actividades definidas en el DCU se pudo obtener la información necesaria para obtener un producto adaptado a las necesidades del usuario.
\end{abstract}

Palabras clave: Diseño centrado en el usuario; diseño participativo; discapacidad intelectual; habilidades matemáticas, educación especial.

\section{Introducción}

Actualmente, el paradigma para referirse a las personas con discapacidad ha cambiado hacia ver a la persona a partir de su funcionalidad en lugar de su deficiencia, motivo por el cual se promueve su inclusión tanto educativa como laboral.

La discapacidad intelectual es una condición en la que una persona presenta una limitación en su coeficiente intelectual con un valor menor a 70, además se acompaña de dificultades adaptativas, como las habilidades de la vida cotidiana, la comunicación y la socialización [1]. En las personas con discapacidad intelectual, entre ellos la población con Síndrome de

\footnotetext{
Durán, AC., Marín, MA., Rocha, KN., Cárdenas, J., González, AL. Facultad de Ingeniería Mecánica y Eléctrica

Universidad Autónoma de Nuevo León

San Nicolás de los Garza, N. L., México.

Email: abies.duranlu@uanl.edu.mx, mario.marinba@uanl.edu.mx, karla.rocharo@uanl.edu.mx, julian.cardenasprz@uanl.edu.mx, aida.gonzalezlr@uanl.edu.mx
}

Down, para lograr la inclusión es importante el aprendizaje y desarrollo de habilidades matemáticas que les permitirán resolver problemas cotidianos, participar responsablemente en la dinámica familiar y social, así como pertenecer a un ambiente laboral o educativo inclusivo.

\subsection{Definición del Problema}

De acuerdo con la Encuesta Nacional de la Dinámica Demográfica (ENADID) 2014, el $6 \%$ de los habitantes de México cuentan con alguna discapacidad y del total de personas con discapacidad, el $38.8 \%$ tiene discapacidad intelectual como se puede observar en la Figura 1 [6].

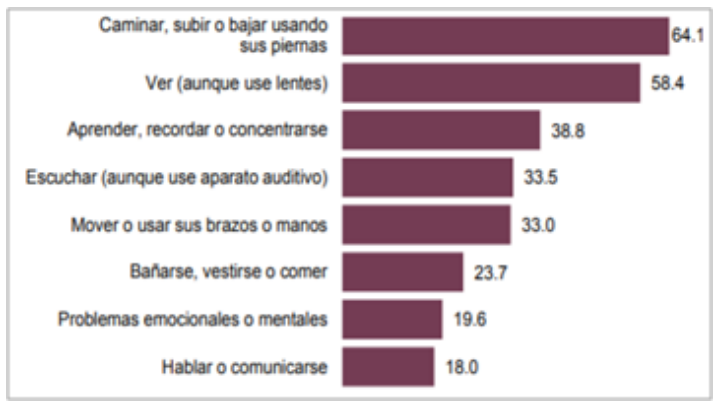

Figura 1. Porcentajes de población con discapacidad, por tipo de discapacidad.

TEDI A.C. (Taller de Expresión y Desarrollo Integral) es un centro reconocido en el estado de Nuevo León, dedicado a la formación y educación de personas con Síndrome de Down, su propósito es elevar su calidad de vida y desarrollar su potencial para que logren su autonomía e integración familiar, educativa y social [9].

El equipo pedagógico de TEDI ha desarrollado una Metodología de Enseñanza-Aprendizaje de Habilidades Matemáticas específicamente para la población con discapacidad intelectual, esta metodología se divide en seis áreas: Prenumérico, Numérico, Manejo de Tiempo, Manejo de Dinero, Geometría y Mediciones.

Las instituciones de educación especial, como TEDI, refieren que no cuentan con material educativo computacional para desarrollo de habilidades matemáticas adaptado que les permita complementar sus programas de educación especial, actualmente 
utilizan aplicaciones comunes las cuales no son acordes a las necesidades de sus alumnos.

Por lo tanto, es importante proponer una solución que ayude a este grupo vulnerable a desarrollar las habilidades para lograr una inclusión de calidad en la sociedad.

\subsection{Justificación del trabajo}

El uso de las Tecnologías de la Información (TI) en la educación para personas con discapacidad intelectual ha permitido realizar adaptaciones, de acuerdo con sus características, para un uso adecuado y permitir el acceso a la información para su formación; un sistema educativo para personas con discapacidad intelectual necesita una interacción y transmisión de conocimiento, y de una interfaz especial para adaptarse a las características y necesidad de este tipo de usuarios [5].

La literatura especializada, ha registrado diversas experiencias e investigaciones sobre el uso de recursos informáticos en el campo de la Educación Especial mediante los que se han evidenciado las posibilidades que brindan estos medios para los procesos de enseñanza y aprendizaje de los alumnos con necesidades educativas especiales tales como: facilidad para interactuar con objetos y personas, mejor expresión de ideas, rol más activo y productivo en sus procesos de aprendizaje [8].

En las escuelas de educación especial, como TEDI, utilizan aplicaciones de aprendizaje de matemáticas no adaptadas a esta población, por lo que su uso es complicado para ellos, ya que las repeticiones no son suficientes y el incremento de complejidad no es adecuado para sus necesidades de aprendizaje.

\section{Propuesta de Solución}

Considerando que el fin último de la educación es asegurar que todos los estudiantes aprendan, potenciando el desarrollo de sus habilidades y destrezas, así como respetando sus diferencias individuales, y frente al gran desafío de acercar el aprendizaje de las matemáticas a todos los estudiantes del país con necesidades educativas especiales, se decidió realizar un trabajo colaborativo uniendo talentos y voluntades en bien de la comunidad para realizar el desarrollo de una aplicación multiplataforma dirigida a personas con discapacidad intelectual que permita potencializar su aprendizaje de habilidades matemáticas mediante el uso de las TI.

Esta aplicación se denominó HIPO (Habilidades Integrales del Pensamiento) y comprende actividades de aprendizaje basadas en una Metodología de Enseñanza-Aprendizaje de Habilidades Matemáticas específicamente creada para la población con discapacidad intelectual por un equipo pedagógico de TEDI., pretende ser un recurso didáctico multimedia y gratuito para la enseñanza de las matemáticas.

\section{Metodología}

Para lograr obtener un producto fácil de usar y que se adapte las necesidades especiales de los usuarios finales de nuestra aplicación utilizamos la Metodología de Diseño Centrado en el Usuario (DCU) y Diseño Participativo. El DCU es una aproximación al diseño de productos y aplicaciones que sitúa al usuario en el centro de todo el proceso, se puede entender como una filosofía cuya premisa es que, para garantizar el éxito de un producto, hay que tener en cuenta al usuario en todas las fases del proceso; son consideradas 4 fases principales para el procedimiento de la metodología las cuales son: Análisis del contexto de uso, Diseño, Prototipo y Evaluación [7].

\subsection{Análisis de contexto de uso y requerimientos}

Se realizó una reunión con la Lic. Alicia Flores, Directora de TEDI, y con la Lic. en Educación especial Sonia Hernández para conocer acerca de la escuela TEDI, nos mostraron la visión, la misión, un video institucional y nos explicaron acerca de las características de las personas que atienden y los programas que ofrecen.

Con base en la información obtenida en las juntas se identificaron los requerimientos de la aplicación, los cuales fueron revisados con el personal de TEDI para generar la siguiente lista de requerimientos:

- Aplicación multiplataforma

- Actividades divididas en las seis áreas del programa curricular

- Instrucciones claras de lo que se debe hacer en cada actividad (en texto y voz)

- Interfaz de usuario sencilla, sin distracciones

- Consistencia en el estilo de diseño e interacción

- Utilizar estímulo de respuesta correcta

- Usar voces variadas de hombre, mujer, niño y niña

Para conocer el contexto de uso, fue necesario asistir a la escuela TEDI, en donde pudimos observar a los niños en sus grupos mientras tomaban clase y posteriormente, como utilizaban de manera individual sus tabletas para identificar qué características serían necesarias en nuestra aplicación.

Durante esta visita registramos las observaciones en formatos y grabamos video para un posterior análisis, esta visita fue de gran valor ya que nos permitió conocer la manera de interactuar y características de comportamiento de esta población que no conocíamos.

Después de la visita nos reunimos para compartir y analizar la información recopilada para lo que construimos un diagrama de afinidad, ver Figura 2; con base en este diagrama se generó una lista de recomendaciones la cual fue revisada con una profesora de TEDI para revisar la interpretación de nuestras observaciones.

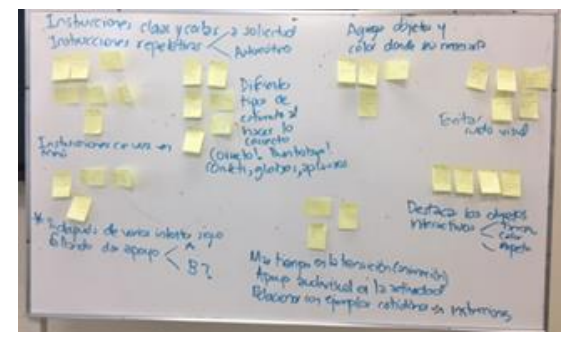

Figura 2. Diagrama de afinidad de observaciones de la visita a TEDI

\subsection{Diseños y prototipado}

\subsubsection{Prototipos de baja fidelidad}

Para las primeras actividades realizamos prototipos de papel con base en las especificaciones de texto recibidas de los profesores de TEDI, en la Figura 3 se puede observar el ejemplo de una de las actividades. Con estos prototipos se hicieron evaluaciones para comprobar que la comprensión de la actividad fue correcta y cumplía el objetivo de la actividad indicada. 


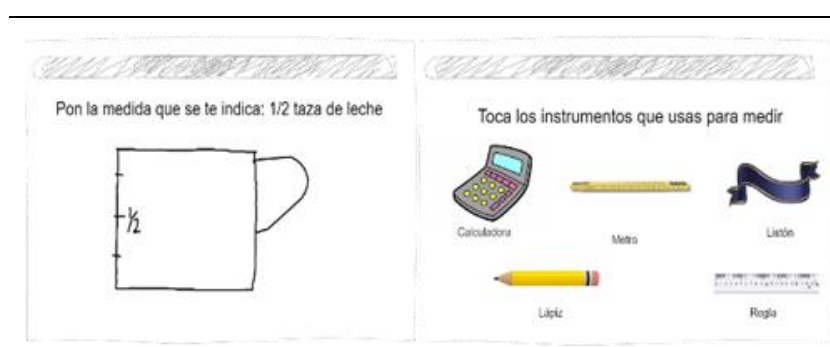

Figura 3. Prototipos de baja fidelidad del área de mediciones.

\subsubsection{Diseño participativo}

En el diseño participativo se cuenta con un taller en el que los desarrolladores, representantes del cliente y usuarios trabajan conjuntamente para diseñar una solución [3].

En nuestro proyecto, la Lic. en Educación Especial Sonia Hernández, autora del programa curricular de matemáticas para personas con discapacidad intelectual y docente de TEDI, fue integrada como un miembro más del equipo de trabajo.

Inicialmente, la Lic. Sonia entregaba las actividades a desarrollar en la aplicación en formato de texto, lo cual ocasionaba dudas al querer plasmarlo en un prototipo, ante esto, se optó por enseñarle a usar el software Cacoo para desarrollar prototipos de baja fidelidad y que de esta manera especificara las actividades. En la Figura 4 se puede observar la comparación entre la misma actividad con solo texto y con el uso del software.

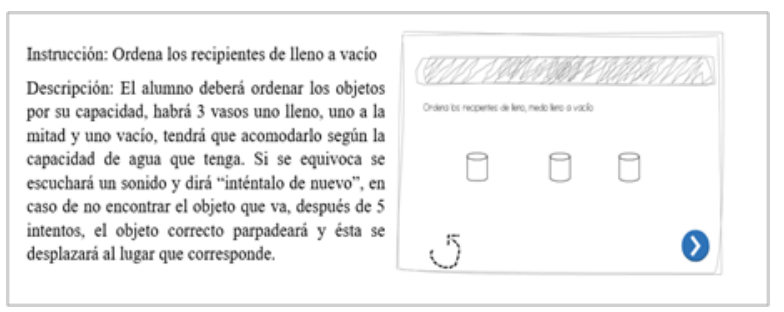

Figura 4. Comparación de actividades de texto y con el software Cacoo.

Para las actividades posteriores, la Lic. Sonia realizó la definición de las actividades mediante prototipos y se realizaba una junta semanal, en la cual el equipo consultaba las dudas de las actividades que se recibían, en la Figura 5 se muestra el uso de los prototipos de las actividades para revisarlas con la Lic. Sonia.

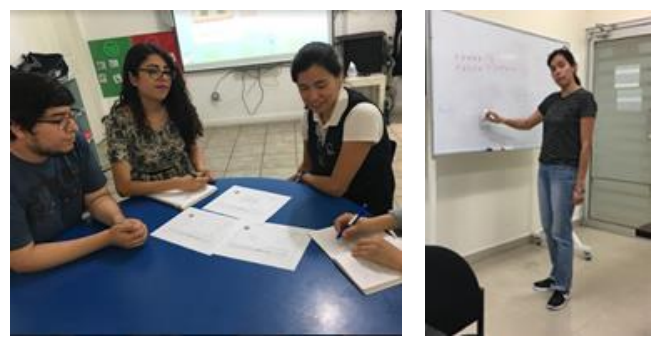

Figura 5. Reunión de revisión con la Lic. Sonia Hernández

Entre las observaciones realizadas durante la visita se identificó que las instrucciones que daban los profesores a los alumnos tenían características como ser claras, cortas y con cierto tono de autoridad y de esa manera eran atendidas por los estudiantes, por lo que se invitó a los profesores de TEDI, Katherine Cueto y Daniel Sánchez a que grabaran los audios de las instrucciones, puesto que ellos cuentan con experiencia de cómo dar instrucciones a niños con discapacidad intelectual además de revisar la claridad para su comprensión. En la Figura 6 se puede observar una sesión de grabación de los audios.

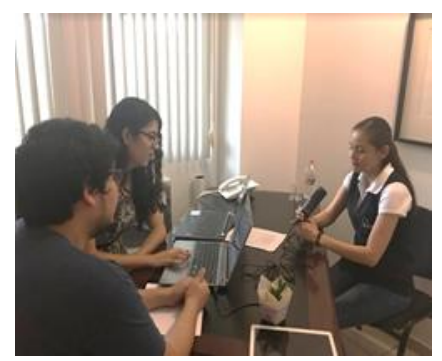

Figura 6. Profesora grabando los audios de la aplicación.

\subsubsection{Diseño plano (flat design)}

El diseño plano o flat design parte de la idea de suprimir o reducir todo tipo de decoración en un diseño de interfaz y así facilitar la funcionalidad [4].

Con lo anterior y tomando en cuenta el perfil de usuario al que se dirige la aplicación, se decidió que el diseño plano se ajustaba a las necesidades de la aplicación debido a la claridad y simplicidad con la que se da el mensaje en cada imagen suprimiendo todo el ruido visual sin tener alguna distracción para el usuario.

Se decidió que todas las imágenes a usar en la aplicación fueran diseñadas por el propio equipo de trabajo, es decir, todas son imágenes originales. En cuanto a la creación de los escenarios o fondos se basó en la descripción de las actividades y de la temática que se eligió, la cual fue "parque de diversiones", en la Figura 7 se puede observar una pantalla con este diseño, esta temática fue definida en conjunto con los profesores de TEDI, con base en los elementos de interés observados en esta población.

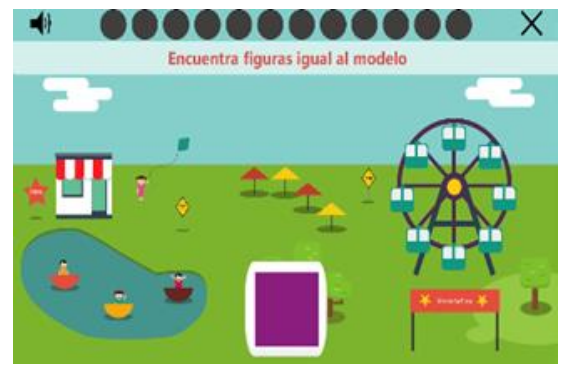

Figura 7. Actividad de área pre-numérico

\subsubsection{Persona}

La técnica persona nos provee permite conocer acerca de cómo los usuarios se comportan, como piensan, que es lo que piensan realizar y el por qué; no son personas verdaderas, pero están basadas en los comportamientos y motivaciones de las personas reales que hemos observado durante el proceso de diseño [2]. Consideramos que el uso de la técnica persona sería muy útil para el desarrollo de la aplicación ya que nos daría una visión amplia para poder satisfacer la necesidad de los usuarios y nos ayudó a mantener en mente las necesidades y elementos adecuados para el usuario durante el diseño y desarrollo, en la Figura 8 se puede observar un fragmento de la persona que creamos. 


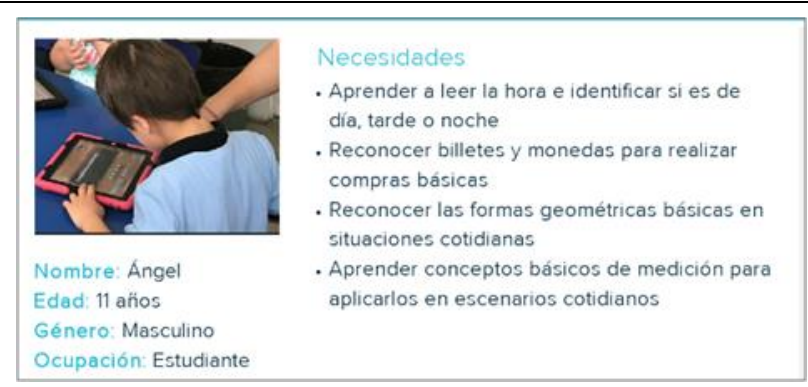

Figura 8. Fragmento de la Persona creada para el diseño.

\subsection{Evaluación}

Se realizaron sesiones de evaluación individuales con los usuarios usando prototipos funcionales, posteriormente se hicieron evaluaciones con prototipos de alta fidelidad en los pizarrones electrónicos y tabletas de TEDI, en este proceso se llenaron formatos de evaluación y se grabó en video el desarrollo de las pruebas con las tareas asignadas, en la Figura 9 se pueden observar los procesos mencionados.
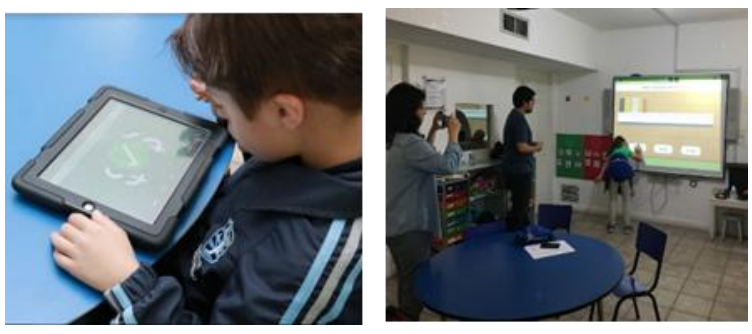

Figura 9. Evaluaciones con los alumnos de TEDI.

Estas evaluaciones nos ayudaron a tener retroalimentación para mejorar la aplicación en donde veíamos las posibles mejoras, los errores, así como una mejor visión para entender la estrategia de aprendizaje que les ayuda más en la cual conforme pasaron las evaluaciones fuimos comprendiendo y los errores se fueron reduciendo.

\subsubsection{Pruebas de seguimiento ocular (Eye-tracker)}

Debido al perfil del usuario, se complicó que pudiera comunicar lo que pensaba acerca del uso de la interfaz, motivo por el cual se tomó la decisión de llevar a cabo pruebas con Eye-tracker o también llamado seguimiento ocular.

Estas pruebas consisten en realizar un monitoreo de la mirada de un usuario hacia imagen, permite mostrar el área, tiempo y orden que fija la atención y tiene como objetivo estudiar la manera en que el usuario explora visualmente la interfaz gráfica del sistema con el que interactúa [10].

En la Figura 10 se puede observar la realización de la prueba con un usuario, en esta se hace un monitoreo de la mirada y nos indica los lugares en donde los usuarios ven realmente antes de llegar al objetivo, también se muestra un mapa de calor del software que muestra el seguimiento ocular.
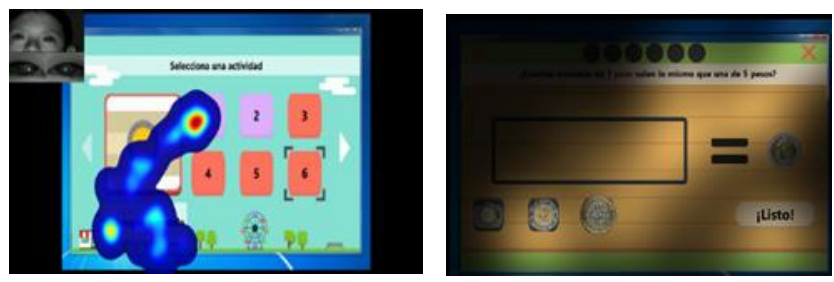

Figura 10. Usuario final realizando pruebas con el eyetracker.
Los resultados del análisis de los usuarios participantes en el seguimiento ocular coincidieron en que ellos tenían su mayor atención hacia los objetos, en la Figura 10 se observa que en la actividad "Equivalencias billetes/monedas" la mayor parte de atención de los usuarios fijaba hacia las monedas, mientras que otros miraban el botón de "Listo" y la sección de la instrucción con la cantidad con lo que podíamos identificar la atención visual del usuario en cada interfaz.

\subsubsection{Evaluaciones de profesores}

Al tener una versión estable de nuestra aplicación se instaló en algunas de las tabletas (iOS) y pizarrones interactivos de TEDI, con la finalidad de que la aplicación pueda ser usada por sus estudiantes y que los profesores hagan una evaluación de la interacción con la aplicación en el contexto de uso, se proporcionó un formato a los profesores para que realizaran el registro de sus observaciones y sugerencias, el cual se puede observar en la Figura 11.

Posteriormente se realizó una junta en la que revisamos todas las observaciones de los profesores y de acuerdo con estas se realizaron cambios y mejoras a la aplicación.

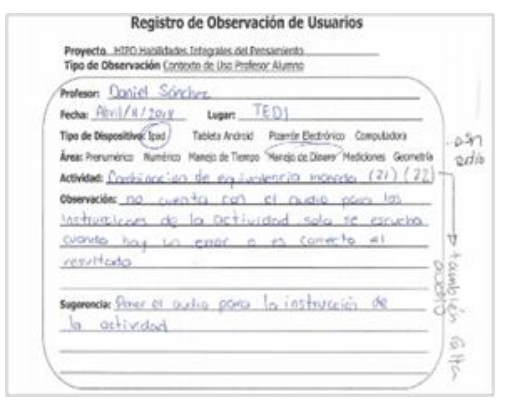

Figura 11. Registro de observación de usuario del profesor Daniel Sánchez.

\section{Resultados}

Se desarrolló HIPO, aplicación que cuenta con 6 áreas para trabajar: conceptos pre-numéricos, número, mediciones, manejo de tiempo y manejo de dinero; áreas prácticas y funcionales para cualquier persona con discapacidad intelectual; podrá utilizarse en plataformas web, PC y dispositivos móviles, tanto en iOS como Android. Las actividades de aprendizaje que contiene son acordes con un programa curricular desarrollado para la población con discapacidad intelectual.

\section{Conclusiones}

Aplicando la Metodología DCU logramos que la aplicación fuera fácil de usar y comprender por el usuario final ya que con las diferentes evaluaciones pudimos identificar áreas de oportunidad y solucionarlas durante el proceso.

La aplicación tiene beneficios ya que existen zonas del país en las cuales no se cuenta con personas preparadas en Educación Especial y este recurso podrá ser utilizado por profesores para apoyar a alumnos con este tipo de limitaciones; además, podrá ser utilizado por los padres de familia para continuar ejercitando las habilidades de sus hijos en el hogar, ya que es conocido que este tipo de población requiere de estímulo constante para incrementar su desarrollo. El desarrollo de estas habilidades contribuirá a que un mayor número de personas de esta población se incluya en entornos educativos y laborales. 


\section{Agradecimientos}

Le agradecemos a todo el personal del Instituto de TEDI por su ayuda y apoyo en el desarrollo de la aplicación HIPO.

\section{Referencias}

[1] AAIDD, Discapacidad Intelectual. Definición, Clasificación Y Sistemas De Apoyo Social Alianza Editorial, (2011).

[2] Cooper, A., Reinmann, R., Cronin, D. About face the essentials of interaction design (4ta. Ed.), John Wiley \& Sons Inc. (2014).

[3] Gaffney, G., Usability Techniques series: Participatory design workshops. Information \& Design, COMMUNICATTIONS OF THE ACM, (2010).

[4] García, J., "Qué es el flat design o Diseño Plano," in Departamento de Internet. Recuperado de: http://www.departamentodeinternet.com/que-es-flat-designdiseno-plano/

[5] González, C.,(et al), Diseño e implementación de interfaces accesibles para acercar las matemáticas a niños con síndrome de Down. In Proc. ICTE.(2006).1-7.
[6] Instituto Nacional de Estadística y Geografía. 2014. La discapacidad en México, datos al 2014 / INEGI.

Aguascalientes, México. Recuperado de: http://internet.contenidos.inegi.org.mx/contenidos/productopr od_serv/contenidos/espanol/bvinegi/productos/nueva_estruc/ 702825090203.pdf

[7] Moreno, L., Martínez, P., Ruiz, B., AIPO, Recuperado de: http://aipo.es/articulos/4/21.pdf

[8] Ramírez, D. A., Giraldo, L. E., \& Henao, O., Diseño y experimentación de una propuesta didáctica apoyada en tecnología multimedial para el desarrollo de habilidades comunicativas en niños con síndrome de down. Revista Educación y Pedagogía, (2015), 11,23, 245-261.

[9] TEDI, Recuperado de: http://tedi.org.m

[10] Yusef, H. M., Solana, V.H, Eye-Tracking en Interacción Persona-Ordenador. No solo usabilidad. Recuperado de: http://www.nosolousabilidad.com/articulos/eye-tracking.htm 